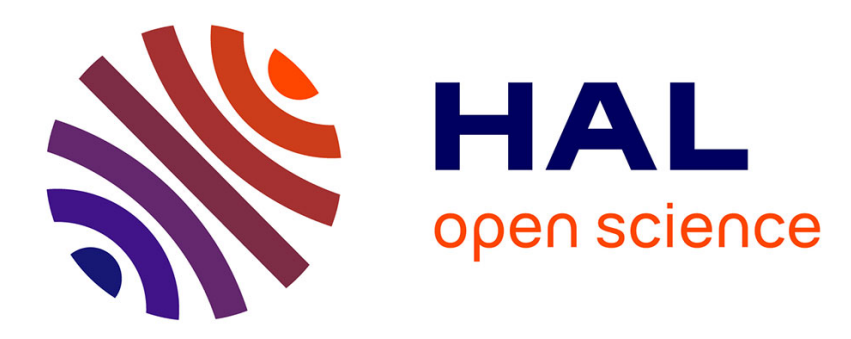

\title{
n-Refined Neutrosophic Groups II
}

Mohammad Abobala

\section{To cite this version:}

Mohammad Abobala. n-Refined Neutrosophic Groups II. International Journal of Neutrosophic Science, 2019, pp.47 - 56. 10.5281/zenodo.3929792 . hal-02915735

\section{HAL Id: hal-02915735 \\ https://hal.science/hal-02915735}

Submitted on 20 Aug 2020

HAL is a multi-disciplinary open access archive for the deposit and dissemination of scientific research documents, whether they are published or not. The documents may come from teaching and research institutions in France or abroad, or from public or private research centers.
L'archive ouverte pluridisciplinaire HAL, est destinée au dépôt et à la diffusion de documents scientifiques de niveau recherche, publiés ou non, émanant des établissements d'enseignement et de recherche français ou étrangers, des laboratoires publics ou privés. 


\title{
n-Refined Neutrosophic Groups II
}

\author{
Mohammad Abobala, Faculty of Science, Tishreen University, Lattakia, Syria \\ e-mail: $\underline{\text { mohammadabobala777@gmail.com }}$
}

\begin{abstract}
The objective of this paper is to study some of AH-substructures in n-refined neutrosophic group. Also, it deals with some elementary properties of AH-subgroups, AH-normality, AH-homomorphisms, and endomorphisms especially in a non abelian n-refined neutrosophic group.
\end{abstract}

Keywords: n-Refined neutrosophic group, AH- subgroup, AH- homomorphism, AH-endomorphism.

\section{Introduction}

Neutrosophy as a new kind of logic founded by F. Smarandache deals with indeterminacy in nature and reality. In particular, it provided a strong tool to study some algebraic structures. Many neutrosophical algebraic structures came to light such as neutrosophic groups, neutrosophic rings, and neutrosophic semi groups. See [2,3,6]. Many studies were carried out using the idea of splitting the indeterminacy I into two components $I_{1}, I_{2}$, like refined neutrosophic groups. See [3]. It has some interesting applications in soft computing [10].

In $[7,8], \mathrm{F}$. Smarandache came with a new idea which suggests the splitting of indeterminacy $I$ into $\mathrm{n}$ subindeterminacies $I_{1}, I_{2}, \ldots, I_{n}$, which refers to many different degrees of indeterminacy. In [9], Smarandache defined algebraic operations between refined neutrosophic numbers and refined neutrosophic sets.

In [1], Abobala has defined the concept of n-refined neutrosophic groups to generalize the classical concept of neutrosophic group. Some interesting notions were presented such as n-refined neutrosophic subgroup, n-refined neutrosophic homomorphism, and AH-subgroup of an n-refined neutrosophic group.

In this work, we continue the work began in [1]. We establish the concept of AH-homomorphism to study AHsubgroups. Also, we handle some related concepts such as AH-nilpotency, AH-solvability, and AHhomomorphisms.

\section{Motivation}

This article is a continuation work of the work began in [1]. It establishes the algebraic theory of some AHsubstructures in an n-refined neutrosophic ring.

\section{Preliminaries}

In the following section, we recall some important and useful definitions about neutrosophic groups and n-refined neutrosophic groups.

Definition 2.1: [2]

DOI: 10.5281/zenodo.3929792 
Let $\left(\mathrm{G},{ }^{*}\right)$ be a group . Then the neutrosophic group is generated by $\mathrm{G}$ and I under * denoted by

$\mathrm{N}(\mathrm{G})=\{<G \cup I>, *\}$.

$I$ is called the indeterminate (neutrosophic element) with the property $I^{2}=I$.

\section{Definition 2.2: [1]}

Let $(G, *)$ be a group, we define the corresponding n-refined neutrosophic group $N_{n}(G)$ as follows:

$N_{n}(G)=\left(<G \cup\left\{I_{1}, \ldots, I_{n}\right\}>, *\right)=\left\{\left(a_{0}, a_{1} I_{1}, \ldots, a_{n} I_{n}\right) ; a_{i} \in G\right\}$.

It is easy to see that $N_{n}(G)$ is closed under $*$, and it is a semi group but not a group since $I_{i}$ has no inverse with respect to $*$ in general.

\section{Remark 2.3: [1]}

If $(\mathrm{G},+)$ is an additive abelian group, then addition on $N_{n}(G)$ can be described as follows:

Consider $x=\left(a_{0}, a_{1} I_{1}, \ldots, a_{n} I_{n}\right), y=\left(b_{0}, b_{1} I_{1}, \ldots, b_{n} I_{n}\right)$, we have

$x+y=\left(a_{0}+b_{0},\left[a_{1}+b_{1}\right] I_{1}, \ldots,\left[a_{n}+b_{n}\right] I_{n}\right)$. In this case $\left(N_{n}(G),+\right)$ is a classical abelian group.

The identity element is $(0,0, \ldots, 0)$.

It is easy to see that $N_{n}(G) \cong G \times G \times . . \times G(n+1$ times $)$ in the case of abelian additive group G.

\section{Remark 2.4: [1]}

If $\mathrm{G}$ is a multiplicative group, then group product on $N_{n}(G)$ can be described as follows:

Consider $x=\left(a_{0}, a_{1} I_{1}, \ldots, a_{n} I_{n}\right), y=\left(b_{0}, b_{1} I_{1}, \ldots, b_{n} I_{n}\right)$, we have

$x y=\left(t_{0}, t_{1}, \ldots, t_{n}\right) ; t_{s}=\prod_{i, j=0}^{n}\left(a_{i} b_{j}\right) I_{i} I_{j} ; I_{0}=e_{G}$ and $I_{i} I_{j}=I_{s}$.

The identity element is $\left(e_{G}, e_{G} I_{1}, \ldots, e_{G} I_{n}\right)$.

In this case $N_{n}(G)$ is not isomorphic to the direct product of $\mathrm{n}+1$ copies of $\mathrm{G}$, since it is not a classical group in this case.

The binary operation between the sub-indeterminacies is $I_{i} \cdot I_{j}=I_{\min (i, j)}$.

\section{Definition 2.5: [1]}

Let $N_{n}(G)=\left\{\left(a_{0}, a_{1} I_{1}, \ldots, a_{n} I_{n}\right) ; a_{i} \in G\right\}$ be an n-refined neutrosophic group,

$N_{n}(H)=\left\{\left(b_{0}, b_{1} I_{1}, \ldots, b_{n} I_{n}\right) ; b_{i} \in H_{i} ; H_{i}\right.$ is a subgroup of $G$ for all $\left.i\right\}$ is called an AH-subgroup of $N_{n}(G)$.

If $H_{i} \cong H_{j}$ for all $i \neq j$, then it is called an AHS-subgroup.

The AH-subgroup $N_{n}(H)$ is called AH-abelian if $H_{i}$ is abelian for all $i$. Also, it is called AH-cyclic if $H_{i}$ is cyclic for all $i$.

\section{Example 2.6: [1]}


Let $G=S_{3}$ be the non abelian symmetric group of order 6, there are two non isomorphic subgroups of G, $K \cong Z_{2}, S \cong Z_{3}$, consider the corresponding 3-refined neutrosophic group $N_{3}(G)$, we have:

$N_{3}(H)=\left(K, S I_{1}, K I_{2}, S I_{3}\right)=\left\{\left(a, b I_{1}, c I_{2}, d I_{3}\right) ; a, c \in K\right.$ and $\left.b, d \in S\right\}$ is an AH-subgroup of $N_{3}(G)$.

$N_{3}(H)$ is an AH-cyclic, since $K, S$ are cyclic.

\section{Definition 2.7: [5]}

Let $\mathrm{G}$ be a group with the following normal series $\{e\}=H_{0} \leq H_{1} \leq \cdots \leq H_{n}=G$. It is called solvable if $H_{i} / H_{i-1}$ is abelian.

The intersection, direct product, and product of two solvable groups is solvable.

Definition 2.8: [11]

(a) Let $\mathrm{G}$ be any group. It is called meta abelian if it has a abelian derivative subgroup $G^{\prime}$.

(b) Let $\mathrm{G}$ be a group. It is called nilpotent if it has a central series.

For the concept of central series, see [11].

$S_{3}$ is a solvable group, but it is not nilpotent.

$D_{4}$ is a meta abelian and nilpotent group.

The intersection, and the direct product of two meta abelian groups is meta abelian.

The intersection, and the direct product of two nilpotent groups is nilpotent.

Definition 2.9: [2]

Let $\mathrm{N}(\mathrm{G})$ be a neutrosophic group and $\mathrm{H}$ be a neutrosophic subgroup, i.e ( $\mathrm{H}$ contains a proper subgroup of $\mathrm{G}$ ) of $\mathrm{N}(\mathrm{G})$. Then $\mathrm{H}$ is a neutrosophic normal subgroup of $\mathrm{N}(\mathrm{G})$ if $x H=H x$ for all $x \in N(G)$.

\section{Definition 2.10: [2]}

Let $N(G)$ be a neutrosophic group. Then the center of $N(G)$ is denoted by $C(N(G))$, and defined $C(N(G))=\{x \in N(G) ; x y=y x \forall y \in N(G)\}$.

\section{Definition 2.11: [2]}

Let $\mathrm{N}(\mathrm{G}), \mathrm{N}(\mathrm{H})$ be two neutrosophic groups, then $N(G) \times N(H)=\{(g, h) ; g \in N(G), h \in N(H)\}$.

Definition 2.12: [6]

Let $\mathrm{N}(\mathrm{G}), \mathrm{N}(\mathrm{H})$ be two neutrosophic groups and $\varphi: N(G) \rightarrow N(H)$ is called a neutrosophic homomorphism if it is a homomrphism between $\mathrm{G}, \mathrm{H}$ and $\varphi(I)=I^{\prime}$.

Where $I^{\prime}$ is the neutrosophic element of $\mathrm{N}(\mathrm{H})$. 
If $\varphi$ is a correspondence one-to-one it is called a neutrosophic isomorphism.

\section{Main discussion}

\section{Definition 3.1:}

Let $\mathrm{G}$ be any group, $N_{n}(G)$ be its corresponding n-refined neutrosophic group,

$N_{n}(H)=\left(H_{0}, H_{1} I_{1}, \ldots, H_{n} I_{n}\right) ; H_{i} \leq G$ be an AH-subgroup of $N_{n}(G)$. We say

(a) $N_{n}(H)$ is AH-normal subgroup if $H_{i}$ is normal for all $i$.

(b) $N_{n}(H)$ is AH-nilpotent subgroup if $H_{i}$ is nilpotent for all $i$.

(c) $N_{n}(H)$ is AH-solvable subgroup if $H_{i}$ is solvable for all $i$.

(d) $N_{n}(H)$ is AH-meta abelian subgroup if $H_{i}$ is meta abelian for all $i$.

(e) $N_{n}(H)$ is AH-simple subgroup if $H_{i}$ is simplefor all $i$.

\section{Example 3.2:}

Consider the symmetric group of order $6\left(G=S_{3}\right)$, it has one normal subgroup $H \cong Z_{3}$, and three 2-Sylow subgroups $K \cong S \cong L \cong Z_{2}$.

Let $N_{2}(G)$ be the corresponding 2-refined neutrosophic group, we have

(a) $M_{1}=\left(K, K I_{1}, H I_{2}\right)$ is an AH-subgroup of $N_{2}(G)$.

(b) $M_{1}$ is an $\mathrm{AH}-$ nilpotent/AH-solvable, since $\mathrm{K}, \mathrm{H}$ are nilpotent, and solvable subgroups of $\mathrm{G}$.

(c) $M_{1}$ is an AH-simple, since $\mathrm{K}, \mathrm{H}$ are simple.

(d) $M_{1}$ is not an AH-normal, since $\mathrm{K}$ is not normal.

(e) $M_{1}$ is an AH-meta abelian, since $\mathrm{H}, \mathrm{K}$ are meta abelian subgroups.

\section{Remark 3.3:}

If $\mathrm{G}$ is an additive abelian group, then any AH-subgroup is a classical subgroup, since $N_{n}(G)$ is a classical group and isomorphic to the direct product of $\mathrm{G}$ with itself ( $\mathrm{n}+1$ times).

\section{Definition 3.4:}

Let G,K be any two groups, $N_{n}(G), N_{n}(K)$ be their corresponding n-refined neutrosophic groups, $f_{i}: G \rightarrow K ; 0 \leq i \leq n$ be a classical homomorphism for all $i$. We say

(a) $f: N_{n}(G) \rightarrow N_{n}(K) ; f\left(a_{0}, a_{1} I_{1}, \ldots, a_{n} I_{n}\right)=\left(f_{0}\left(a_{0}\right), f_{1}\left(a_{1}\right) I_{1}, \ldots, f_{n}\left(a_{n}\right) I_{n}\right)$ is an AH-homomorphism.

(b) If $f_{i}: G \rightarrow K$ is an isomorphism for all $i$, then $f: N_{n}(G) \rightarrow N_{n}(K)$ is called an AH-isomorphism.

(c) If $f_{i}=f_{j} ; i \neq j$, then $f: N_{n}(G) \rightarrow N_{n}(K)$ is called an AHS-homomorphism. 
(d) The AH-kernel is defined as follows: $A H-\operatorname{Ker}(f)=\left(\operatorname{Ker}\left(f_{0}\right), \operatorname{Ker}\left(f_{1}\right) I_{1}, \ldots, \operatorname{Ker}\left(f_{n}\right) I_{n}\right)$.

(e) The AH-image is defined as: $A H-\operatorname{Im}(f)=\left(f_{0}(G), f_{1}(G) I_{1}, \ldots, f_{n}(G) I_{n}\right)$.

We denote to the AH-homomorphism : $N_{n}(G) \rightarrow N_{n}(K) ; f\left(a_{0}, a_{1} I_{1}, \ldots, a_{n} I_{n}\right)=\left(f_{0}\left(a_{0}\right), f_{1}\left(a_{1}\right) I_{1}, \ldots, f_{n}\left(a_{n}\right) I_{n}\right)$ by $f=\left(f_{0}, f_{1} I_{1}, \ldots, f_{n} I_{n}\right)$.

\section{Definition 3.5:}

Let $\mathrm{G}$ be any group, $N_{n}(G)$ be its corresponding n-refined neutrosophic group, $N_{n}(H)=\left(H_{0}, H_{1} I_{1}, \ldots, H_{n} I_{n}\right), N(K)=\left(K_{0}, K_{1} I_{1}, \ldots, K_{n} I_{n}\right) ; H_{i}, K_{i} \leq G$ be any two AH-subgroups of $N_{n}(G)$.

(a) We define the intersection as follows: $N_{n}(H) \cap N_{n}(K)=\left(H_{0} \cap K_{0},\left(H_{1} \cap K_{1}\right) I_{1}, \ldots,\left(H_{n} \cap K_{n}\right) I_{n}\right)$.

(b) We define the product as follows: $N_{n}(H) . N_{n}(K)=\left(H_{0} . K_{0},\left(H_{1} \cdot K_{1}\right) I_{1}, \ldots,\left(H_{n} \cdot K_{n}\right) I_{n}\right)$.

(c) We define the direct product as follows: $N_{n}(H) \times N_{n}(K)=\left(H_{0} \times K_{0},\left(H_{1} \times K_{1}\right) I_{1}, \ldots,\left(H_{n} \times K_{n}\right) I_{n}\right)$.

\section{Example 3.6:}

Consider the symmetric group of order $6\left(G=S_{3}\right)$, it has one normal subgroup $H \cong Z_{3}$, and three 2-Sylow subgroups $K \cong S \cong L \cong Z_{2}$.

Let $N_{2}(G)$ be the corresponding 2-refined neutrosophic group, $M_{1}=\left(K, K I_{1}, H I_{2}\right), M_{2}=\left(H, K I_{1}, K I_{2}\right)$ be two AHsubgroups of $N_{2}(G)$, we have

(a) $M_{1} \cap M_{2}=\left(\{e\}, K I_{1},\{e\} I_{2}\right)$, which is an AH-subgroup of $N_{2}(G)$.

(b) $M_{1} \cdot M_{2}=\left(K H, K K I_{1}, H K I_{2}\right)=\left(G, K I_{1}, G I_{2}\right)$.

\section{Example 3.7:}

Let $G=(Z,+), K=\left(Z_{6},+\right)$ be two groups, $N_{2}(G), N_{2}(K)$ be the corresponding 2-refined neutrosophic groups, we have

(a) $f_{0}: G \rightarrow K ; f_{0}(a)=a \bmod 6, f_{1}: G \rightarrow K ; f_{1}(a)=2 a \bmod 6$ are two classical homomorphisms.

(b) $f: N_{2}(G) \rightarrow N_{2}(K) ; f\left(a_{0}, a_{1} I_{1}, a_{2} I_{2}\right)=\left(f_{0}\left(a_{0}\right), f_{0}\left(a_{1}\right) I_{1}, f_{1}\left(a_{2}\right) I_{2}\right)=\left(a_{0} \bmod 6, a_{1} \bmod 6 I_{1}, 2 a_{2} \bmod 6 I_{2}\right)$ is an AH-homomorphism.

(c) $A H-\operatorname{Ker}(f)=\left(\operatorname{Ker}\left(f_{0}\right), \operatorname{Ker}\left(f_{0}\right) I_{1}, \operatorname{Ker}\left(f_{1}\right) I_{2}\right)=\left(6 Z, 6 Z I_{1}, 3 Z I_{2}\right)$.

(d) $A H-\operatorname{Im}(f)=\left(\operatorname{Im}\left(f_{0}\right), \operatorname{Im}\left(f_{0}\right) I_{1}, \operatorname{Im}\left(f_{1}\right) I_{2}\right)=\left(Z_{6}, Z_{6} I_{1},\{0,2,4\} I_{2}\right)$.

\section{Theorem 3.8:}

Let $\mathrm{G}$ be any group, $N_{n}(G)$ be its corresponding n-refined neutrosophic group,

$N_{n}(H)=\left(H_{0}, H_{1} I_{1}, \ldots, H_{n} I_{n}\right), N(K)=\left(K_{0}, K_{1} I_{1}, \ldots, K_{n} I_{n}\right) ; H_{i}, K_{i} \leq G$ be any two AH-subgroups of $N_{n}(G)$. We have:

(a) $N_{n}(H) \cap N_{n}(K)$ is an AH-subgroup of $N_{n}(G)$. 
(b) If $N_{n}(H), N_{n}(K)$ are AH-normal, then $N_{n}(H) \cdot N_{n}(K)$ is AH-normal.

(c) $N_{n}(H) \times N_{n}(K)$ is an AH-subgroup of $N_{n}(G) \times N_{n}(G)$.

(d) If $N_{n}(H), N_{n}(K)$ are AH-abelian, then $N_{n}(H) \cap N_{n}(K), N_{n}(G) \times N_{n}(G)$ are AH-abelian.

(e) If $N_{n}(H), N_{n}(K)$ are AH-cyclic, then $N_{n}(H) \cap N_{n}(K)$ is AH-cycilc.

(f) If $N_{n}(H), N_{n}(K)$ are AH-nilpotent, then $N_{n}(H) \cap N_{n}(K), N_{n}(G) \times N_{n}(G)$ are AH-nilpotent.

(g) If $N_{n}(H), N_{n}(K)$ are AH-solvable, then $N_{n}(H) . N_{n}(K), N_{n}(G) \times N_{n}(G), N_{n}(G) \cap N_{n}(G)$ are AH-solvable.

(h) If $N_{n}(H), N_{n}(K)$ are AH- meta abelian, then $N_{n}(H) \cap N_{n}(K), N_{n}(G) \times N_{n}(G)$ are AH-meta abelian.

Proof:

It is well known from the classical group theoretical properties that $H_{i} \cap K_{i}$ is a subgroup of $\mathrm{G}, H_{i} \times K_{i}$ is a subgroup of $G \times G$, and $H_{i} . K_{i}$ is a subgroup of $\mathrm{G}$ under the assumption of normality of $H_{i}, K_{i}$, thus (a),(b),(c) are true.

Also, the direct product and the intersection of any two abelian, nilpotent, or solvable subgroups is the same, thus

(d),(f),(g) are true.

(h), (e) hold by the same argument.

\section{Theorem 3.9:}

Let G,K be any two groups, $N_{n}(G), N_{n}(K)$ be their corresponding n-refined neutrosophic groups,

$N_{n}(H)=\left(H_{0}, H_{1} I_{1}, \ldots, H_{n} I_{n}\right) ; H_{i} \leq G$ be an AH-subgroup, $f_{i}: G \rightarrow K ; 0 \leq i \leq n$ be a classical homomorphism for all $i, f: N_{n}(G) \rightarrow N_{n}(K) ; f\left(a_{0}, a_{1} I_{1}, \ldots, a_{n} I_{n}\right)=\left(f_{0}\left(a_{0}\right), f_{1}\left(a_{1}\right) I_{1}, \ldots, f_{n}\left(a_{n}\right) I_{n}\right)$ be an AH-homomorphism, we have:

(a) If $N_{n}(H)$ is AH-cyclic/AH-abelian, then $f\left(N_{n}(H)\right)$ is AH-cyclic/AH-abelian.

(b) If $N_{n}(H)$ is AH-nilpotent/AH-solvable, then $f\left(N_{n}(H)\right)$ is AH-nilpotent/AH-solvable.

(c) If $N_{n}(H)$ is AH-meta abelian, then $f\left(N_{n}(H)\right)$ is AH-meta abelian.

(d) If $N_{n}(H)$ is AH-normal, then $f\left(N_{n}(H)\right)$ is AH-normal.

(e) AH-Ker(f) is an AH-normal subgroup of $N_{n}(G)$.

(f) AH-Im(f) is an AHS-subgroup of $N_{n}(K)$.

Proof:

(a),(b),(c),(d) It is well known that the homomorphic image of cyclic, abelian, nilpotent, normal, or meta abelian subgroup is the same, so the proof is complete.

(e) Since $\operatorname{Ker}\left(f_{i}\right)$ is a normal subgroup of $\mathrm{G}$ for all $i, A H-\operatorname{Ker}(f)=\left(\operatorname{Ker}\left(f_{0}\right), \operatorname{Ker}\left(f_{1}\right) I_{1}, \ldots, \operatorname{Ker}\left(f_{n}\right) I_{n}\right)$ as an AH-normal subgroup.

(f) The proof is similar to (e). 


\section{Theorem 3.10:}

Let $\mathrm{G}$ be any finite group, $N_{n}(G)$ be its corresponding n-refined neutrosophic group, $N_{n}(H)=\left(H_{0}, H_{1} I_{1}, \ldots, H_{n} I_{n}\right)$ be any AH-subgroup. Then

(a) $O\left(N_{n}(H)\right)=O\left(H_{0}\right) \times O\left(H_{1}\right) \times \ldots \times O\left(H_{n}\right)$.

(b) Lagrange's theorem is true for AH-subgroups, i.e $O\left(N_{n}(H)\right)$ divides $O\left(N_{n}(G)\right)$.

Proof:

(a) Since $\left.N_{n}(H)=\left(H_{0}, H_{1} I_{1}, \ldots, H_{n} I_{n}\right)=\left(h_{0}, h_{1} I_{1}, \ldots, h_{n} I_{n}\right) ; h_{i} \in H_{i}\right\}$, we get $O\left(N_{n}(H)\right)=O\left(H_{0}\right) \times O\left(H_{1}\right) \times$.. $\times$ $O\left(H_{n}\right)$.

(b) Since $O\left(H_{i}\right)$ is a divisor of $O(G)=m$, then $O\left(N_{n}(H)\right)=O\left(H_{0}\right) \times O\left(H_{1}\right) \times \ldots \times O\left(H_{n}\right)$ is a divisor of $O\left(N_{n}(G)\right)=m^{n+1}$. See $[1]$

\section{Definition 3.11:}

Let $(G,+)$ be any additive abelian group, $N_{n}(G)$ be its corresponding n-refined neutrosophic group, $A H-\operatorname{End}\left(N_{n}(G)\right)$ is defined to be the set of all AH-homomorphisms between $N_{n}(G)$ and itself. $A H S-\operatorname{End}\left(N_{n}(G)\right)$ is defined to be the set of all AHS-homomorphisms between $N_{n}(G)$ and itself.

\section{Definition 3.12:}

Let $(G,+)$ be any additive abelian group, $N_{n}(G)$ be its corresponding n-refined neutrosophic group.

We define operations on $A H-\operatorname{End}\left(N_{n}(G)\right)$ as follows:

Let $f: N_{n}(G) \rightarrow N_{n}(G) ; f\left(a_{0}, a_{1} I_{1}, \ldots, a_{n} I_{n}\right)=\left(f_{0}\left(a_{0}\right), f_{1}\left(a_{1}\right) I_{1}, \ldots, f_{n}\left(a_{n}\right) I_{n}\right)$, $g: N_{n}(G) \rightarrow N_{n}(G) ; g\left(a_{0}, a_{1} I_{1}, \ldots, a_{n} I_{n}\right)=\left(g_{0}\left(a_{0}\right), g_{1}\left(a_{1}\right) I_{1}, \ldots, g_{n}\left(a_{n}\right) I_{n}\right)$ be any two AH-endomorphisms, Addition is defined as $(f+g)\left(a_{0}, a_{1} I_{1}, \ldots, a_{n} I_{n}\right)=\left(\left[f_{0}+g_{0}\right]\left(a_{0}\right),\left[f_{1}+g_{1}\right]\left(a_{1}\right) I_{1}, \ldots,\left[f_{n}+g_{n}\right]\left(a_{n}\right) I_{n}\right)$. Multiplication is defined as $(f \circ g)\left(a_{0}, a_{1} I_{1}, \ldots, a_{n} I_{n}\right)=\left(\left[f_{0} \circ g_{0}\right]\left(a_{0}\right),\left[f_{1} \circ g_{1}\right]\left(a_{1}\right) I_{1}, \ldots,\left[f_{n} \circ g_{n}\right]\left(a_{n}\right) I_{n}\right)$. It is easy to see that addition and multiplication are well defined.

$f+g, f \circ g$ are AH-endomorphisms, since $f_{i}+g_{i}, f_{i} o g_{i}$ are two classical endomorphisms for all $i$.

This means that $A H-\operatorname{End}\left(N_{n}(G)\right)$ is closed under addition and multiplication.

\section{Theorem 3.13:}

Let $(G,+)$ be any additive abelian group, $N_{n}(G)$ be its corresponding n-refined neutrosophic group. Then $\left(A H-\operatorname{End}\left(N_{n}(G)\right),+, o\right)$ is a ring and it is isomorphic to $\operatorname{End}(G,+) \times \operatorname{End}(G,+) \times \ldots \times \operatorname{End}(G,+)(\mathrm{n}+1$ times $)$. Proof: 
Simply, we find that $\left(A H-\operatorname{End}\left(N_{n}(G)\right),+\right)$ is an abelian group.

Also, multiplication is associative and distributive with respect to addition, since it is associative and distributive for each component $i$. Thus $\left(A H-\operatorname{End}\left(N_{n}(G)\right),+, o\right)$ has a structure of ring.

The ring isomorphism between $\left(A H-\operatorname{End}\left(N_{n}(G)\right),+, o\right)$ and

$\operatorname{End}(G,+) \times \operatorname{End}(G,+) \times . . \times \operatorname{End}(G,+)(\mathrm{n}+1$ times $)$ can be defined as follows:

$\varphi: A H-\operatorname{End}\left(N_{n}(G)\right) \rightarrow \operatorname{End}(G,+) \times \operatorname{End}(G,+) \times . . \times \operatorname{End}(G,+)$

$\varphi\left(f_{0}, f_{1} I_{1}, \ldots, f_{n} I_{n}\right)=\left(f_{0}, f_{1}, \ldots, f_{n}\right)$.

\section{Definition 3.14:}

Let $\mathrm{G}$ be any group, $N_{n}(G)$ be its corresponding n-refined neutrosophic group,

$N_{n}(H)=\left(H_{0}, H_{1} I_{1}, \ldots, H_{n} I_{n}\right) ; H_{i} \leq G$ be an AH-subgroup of $N_{n}(G)$. We call $N_{n}(H)$ an AH-p subgroup if $H_{i}$ is a pgroup for all $i$.

Clearly, if $\mathrm{G}$ is a finite group, then $O\left(N_{n}(H)\right)$ is a prime power according to Theorem 3.10 .

\section{Definition 3.15:}

Let $\mathrm{G}$ be any group, $N_{n}(G)$ be its corresponding n-refined neutrosophic group,

$N_{n}(H)=\left(H_{0}, H_{1} I_{1}, \ldots, H_{n} I_{n}\right) ; H_{i} \leq G$ be an AH-subgroup of $N_{n}(G)$. The AH-derived subgroup of $H$ can be defined as $\left[N_{n}(H)\right]^{\prime}=\left(H_{0}^{\prime}, H_{1}^{\prime} I_{1}, \ldots, H_{n}^{\prime} I_{n}\right) ; H_{i}^{\prime}=\left\langle x^{-1} y^{-1} x y ; \forall x, y \in H_{i}>\right.$.

\section{Theorem 3.16:}

Let $\mathrm{G}$ be any group, $N_{n}(G)$ be its corresponding n-refined neutrosophic group,

If $N_{n}(H)=\left(H_{0}, H_{1} I_{1}, \ldots, H_{n} I_{n}\right) ; H_{i} \leq G$ be any AH-subgroup of $N_{n}(G)$. Then its AH-derived subgroup is trivial if and only if it is an AH-abelian subgroup.

Proof:

Suppose that $N_{n}(H)=\left(H_{0}, H_{1} I_{1}, \ldots, H_{n} I_{n}\right)$ is AH-abelian, hence $H_{i}$ is abelian for all $i$. This implies that $H_{i}^{\prime}$ is trivial, thus $\left[N_{n}(H)\right]^{\prime}=\left(H_{0}^{\prime}, H_{1}^{\prime} I_{1}, \ldots, H_{n}^{\prime} I_{n}\right)$ is trivial.

Conversrly, If $\left[N_{n}(H)\right]^{\prime}=\left(H_{0}^{\prime}, H_{1}^{\prime} I_{1}, \ldots, H_{n}^{\prime} I_{n}\right)$ is trivial, we find that $H_{i}^{\prime}$ is trivial, thus $H_{i}$ is abelian subgroup of G, which means that $N_{n}(H)=\left(H_{0}, H_{1} I_{1}, \ldots, H_{n} I_{n}\right)$ is AH-abelian.

\section{Remark 3.17:}

There is a well known criteria for solvability of any classical group G. This criteria suggests that any group $\mathrm{G}$ is solvable if and only if the normal series formed from derived subgroups $\left(G^{\prime}, G^{\prime \prime}, \ldots\right)$ reaches to the trivial subgroup $\{$ e $\}$ after n-steps.

We can use this argument to determine if an AH-subgroup is AH-solvable or not by computing AH-derivatives 
$\left(\left[N_{n}(H)\right]^{\prime},\left[N_{n}(H)\right]^{\prime \prime}, \ldots\right)$. If the previous series reaches to the trivial subgroup of $N_{n}(G)$, then $N_{n}(H)$ is an AHsolvable subgroup.

Also, any group $\mathrm{G}$ is meta abelian if and only if its derivative is an abelian subgroup. This idea can be generalized into AH-subgroups as follows:

Any AH-subgroup $N_{n}(H)$ is AH-meta abelian if and only if its AH-derivative subgroup is an AH-abelian.

\section{Remark 3.18}

By using the same argument in Remark 3.17, we can define the AH-cenral series to study the AH-nilpotency of and AH-subgroup.

\section{Conclusion}

In this article we have defined the concept of AH-homomorphism in an n-refined neutrosophic group for the first time. Also, we have introduced some corresponding notions such as AH-endomorphism, AH-solvability, AHnilpotency, and other related concepts. Many examples and theorems were constructed to clarify the validity of these concepts.

Funding: "This research received no external funding"

Conflicts of Interest: "The authors declare no conflict of interest."

\section{References}

[1] Abobala, M., " n-Refined Neutrosophic Groups I", International Journal of Neutrosophic Science, submitted 2019.

[2] Agboola, A.A.A,. Akwu, A.D,. and Oyebo, Y.T,. "Neutrosophic Groups and Subgroups", International .J .Math.Combin, Vol. 3, pp. 1-9, 2012.

[3] Agboola, A.A.A., Akinola, A.D., and Oyebola, O.Y.," NeutrosophicRings I ", International J.Mathcombin, Vol. 4, pp. 1-14, 2011.

[4] Agboola, A.A.A., "On Refined Neutrosophic Algebraic Structures", Neutrosophic Sets and Systems, Vol. 10, pp. 99-102, 2015.

[5] Haushi, M., " Algebraic Structures 1", Tishreen University Press, pp. 102-209, 2005.

[6] Kandasamy, V.W.B., and Smarandache, F., "Some Neutrosophic Algebraic Structures and Neutrosophic NAlgebraic Structures", Hexis, Phonex, Arizona 2006.

[7] Smarandache, F., "Symbolic Neutrosophic Theory", EuropaNova asbl, Bruxelles, 2015.

[8] Smarandache, F., "n-Valued Refined Neutrosophic Logic and Its Applications in Physics", Progress in Physics, Vol. 4, pp.143-146, 2013.

[9] Smarandache, F., "Refined Literal Indeterminacy and the Multiplication Law of Sub-Indeterminacies", Neutrosophic Sets ans Systems, Vol. 9, 2015. 
[10]Deli, I., " Refined Neutrosophic Sets and Refined Neutrosophic Soft Sets: Theory and Applications", Hand Book of Research on generalized and Hybrid Set Structures and Applications for Soft Computing, 2016.

[11] Rotman, J., "The Theory of Groups", University of Illinois, Urbana, pp. 120-180, 1971. 